\title{
Management of Midfacial Fractures: An Overview
}

\author{
${ }^{1}$ GK Thapliyal, ${ }^{2} \mathrm{H}$ Ranga Rajan
}

\begin{abstract}
Trauma to the midface involves fracture of the maxilla, the zygoma, orbit and the nasoorbital ethmoid complex. The facial skeleton is made up of thin segments of bone encased and supported by a more rigid framework of 'buttresses'. The midface is anchored to the cranium through this framework. Midfacial fractures can occur in isolation or in combination with other serious injuries. The road traffic accidents are still the main etiology of the midface fractures followed by assault, fall, sports and warfare injuries. The management of the midface remains a challenge for the maxillofacial surgeons, demanding both skill and expertise.

Midface fractures are most often seen in young adult males whereas there is very low incidence in children. There is little difference in the outcome of stable patients whose fractures are treated very early vs those treated in a more delayed fashion. Rigid and semirigid plating systems perform well with few complications when used to repair midfacial fractures provided proper reduction is obtained. The dental occlusion must be used as the lower frame of reference when treating fractures that result in instability of the maxillary alveolus relative to the skull base. The most common complication after rigid repair of facial fractures appears to be malunion secondary to inadequate reduction.
\end{abstract}

Keywords: Midface, Trauma, Zygomatic complex, Open reduction.

How to cite this article: Thapliyal GK, Ranga Rajan H. Management of Midfacial Fractures: An Overview. J Postgrad Med Edu Res 2014;48(2):68-74.

Source of support: Nil

Conflict of interest: None

\section{INTRODUCTION}

Trauma to the midface involves fracture of the maxilla, the zygoma, orbit and the nasoorbital ethmoid complex. Also the fractures of the midface can generally be classified into central and lateral midface fractures. The facial skeleton is made up of thin segments of bone encased and supported by a more rigid framework of 'buttresses' (Horizontal and the vertical buttresses of the facial skeleton). The midface is anchored to the cranium through this framework. The road traffic accidents are still the main etiology of the midface fractures followed by assault, fall, sports and warfare injuries. The management of fractures of the maxillofacial

\footnotetext{
${ }^{1}$ Professor, ${ }^{2}$ Reader

${ }^{1,2}$ Department of Oral and Maxillofacial Surgery, Indian Army Haryana, India
}

Corresponding Author: GK Thapliyal, Professor, Department of Oral and Maxillofacial Surgery, Indian Army, Haryana, India Phone: 0172-26892545, e-mail: thapliyalgk@yahoo.com complex especially the midface remains a challenge for the oral and maxillofacial surgeon, demanding both skill and expertise.

In 2003, Motamedi ${ }^{1}$ reported the distribution of facial fractures as $72.9 \%$ mandibular, $13.9 \%$ maxillary, $13.5 \%$ zygomatic, $24.0 \%$ zygomatico-orbital, $2.1 \%$ cranial, $2.1 \%$ nasal and $1.6 \%$ frontal injuries. Midfacial fractures can occur in isolation or in combination with other injuries. The distribution of maxillary fractures was 54.6\% Le Fort II, 24.2\% Le Fort I, 12.1\% Le Fort III, 9.1\% alveolar. According to Cook and Rowe, ${ }^{2}$ midfacial injuries occur most frequently in individuals aged 21-30 years (43\%). Most (83.1\%) midfacial fractures occur in males, with the remainder (16.9\%) occurring in females.

\section{REVIEW OF LITERATURE}

The earliest known writings of maxillofacial fractures were recorded in the Edwin Smith Papyrus in 1650 BC which were purchased by Smith in 1862 and translated by Breasted. Traditionally healing and religion were closely interwined as illustrated in the Hellenic period at the temple of Asklepios, where priests provided medical care. To one of these priests a son was born in $460 \mathrm{BC}$ named Hippocrates. Hippocrates who is often portrayed as the 'father of medicine' described a myriad of facial injuries in around $400 \mathrm{BC}$ and his insight provided the basis for bandage and single jaw interdental wiring as methods of fixation and stabilization of facial fractures. ${ }^{3}$

Over the subsequent centuries following Hippocrates there appeared many techniques which in essence were variations of his methods. In the nineteenth century Charles Fredrich Reiche provided the first detailed treatise of maxillary fractures. Carl van Graefe reported on the use of an elastic tube placed into the nose to maintain patency of the airway and also described the use of head frame to treat a maxillary fracture. It was also in the same century that Garretson and Blair advocated maxillomandibular wiring with the aid of splints to primarily treat maxillary fractures. In 1901, a French surgeon, Rene' Le Fort, ${ }^{4}$ published his classical paper on midfacial fracture patterns. His study has ever since been the basis for the description of maxillary fractures. In 1906, Lothrop was the first to describe the use of an antrostomy approach to reduce a medially and inferiorly displaced zygoma. In 1909 Keen described an intraoral approach to the zygomatic arch. In 1927 Gillies $^{5}$ described a 
technique to reduce a zygomatic arch as well as manipulate a fractured zygoma. In 1942 Adam utilized direct wiring to obtain better stability of zygomatic fractures. For years his protocol appeared to be the mainstay of treatment at many institutions. In the 1970s osteosynthesis became a reality for facial fractures with the Swiss Arbeitsgemeinschaft für Osteosynthesefragen (Association for the Study of Internal Fixation or AO) developing miniplate fixation. Today, the use of miniplates provides the principal modality of treatment for reduction and fixation of displaced midfacial fractures.

\section{ANATOMY}

The middle third of the facial skeleton is defined as an area bounded superiorly by a line drawn across the skull from the zygomaticofrontal suture, across the frontonasal and frontomaxillary sutures, to the zygomaticofrontal suture on the opposite side; and inferiorly by the occlusal plane of the maxillary teeth, or, in an edentulous patient, by the maxillary alveolar ridge. It extends posteriorly to the frontal bone in the superior region and the body of the sphenoid in the inferior region, and the pterygoid plates of the sphenoid are usually involved in any severe fracture. The severity and pattern of a fracture depend on the magnitude of the causative force, impact duration, the surface area of the impact site, the acceleration imparted by impact to the affected part of the body and the rate of acceleration. The frontal bone, the sphenoid body and greater and lesser wings are not usually fractured. In fact, they are protected to a considerable extent by the cushioning effect achieved as the fracturing force crushes the comparatively weak bones comprising the middle third of the facial skeleton.

\section{PRIMARY MANAGEMENT}

The initial assessment and management of a patient's injuries must be completed in an accurate and systematic manner to quickly establish the extent of any damage to vital life support systems. The primary survey progresses in a logical manner based on the ABC pneumonic: airway maintenance with cervical spine control, breathing and adequate ventilation, and circulation with control of hemorrhage. The letters $\mathrm{D}$ and $\mathrm{E}$ have also been added: a brief neurologic examination to establish the degree of consciousness, and exposure of the patient via complete undressing to avoid overlooking injuries camouflaged by clothing. Neither midfacial fractures nor cerebrospinal rhinorrhea are contraindications to nasal intubation in emergency.

The physical examination should begin with an evaluation of soft-tissue injuries. The eyelids should be elevated to allow evaluation of the eyes for neurologic and ocular damage. The bony landmarks should be palpated, beginning with the supraorbital and lateral orbital rims and followed by the infraorbital rims, malar eminences, zygomatic arches, and nasal bones. Any steps or irregularities along the bony margin are suggestive of a fracture. Numbness over the area of distribution of the nerve is usually noted with fractures of the facial skeleton. The oral cavity should be inspected and evaluated for lost teeth, lacerations, and occlusal alterations. Any tooth lost at the time of injury must be accounted for because it may have been aspirated or swallowed. The neck should also be examined for injury. Carotid pulses should be assessed.

\section{LE FORT FRACTURES}

Rene Le Fort famously characterized the types of midfacial fracture caused by anteriorly directed forces. The basic principle employed in the treatment of Le Fort fractures is fixation of the maxilla to the next highest stable structure, which differs with Le Fort fracture level. At the Le Fort I level, fixation is performed along the vertical buttresses of the maxilla at the piriform and zygomatic buttress. At higher Le Fort levels, fixation to the nasal bones, orbital rims, or zygomaticofrontal sutures may be necessary. The restoration of proper occlusion is a main goal of treatment. Reconstruction and fixation of the paranasal and zygomaticoalveolar buttresses are often sufficient to reestablish the proper position of the maxilla in Le Fort I fractures. Intermaxillary fixation with arch bars should be performed after reduction of the maxilla, followed by internal fixation of the maxillary vertical buttresses with plates and screws.

Le Fort I fractures can generally be approached via maxillary vestibular incisions. Reduction of the maxilla can be challenging because of impaction, telescoping, or a significant interval of time between injury and treatment. If resistance is encountered during mobilization of the maxilla, Rowe and Hayton Williams disimpaction forceps may be used to help reduce the fracture. Incomplete fractures may make maxillary mobilization difficult; in such cases, completion of the fracture with osteotomies can facilitate reduction (Figs 1 to 8). In cases of severe comminution, inadequate dentition, periodontal disease, or edentulous arches (Gunning splints), fabricated occlusal or palatal splints can be applied to establish intermaxillary fixation.

Le Fort II fractures can be reduced with Rowe disimpaction forceps and intermaxillary fixation. A maxillary vestibular incision and any of various approaches to the orbital rim can be used if open reduction is necessary. Bilateral Lynch incisions are to expose the nasofrontal suture.

Le Fort III fractures rarely occur in isolation and are usually components of panfacial fractures (Figs 9 to 16). Bicoronal incisions can be used to expose the nasoorbitoethmoidal region, fronto zygomatic sutures and lateral orbital 


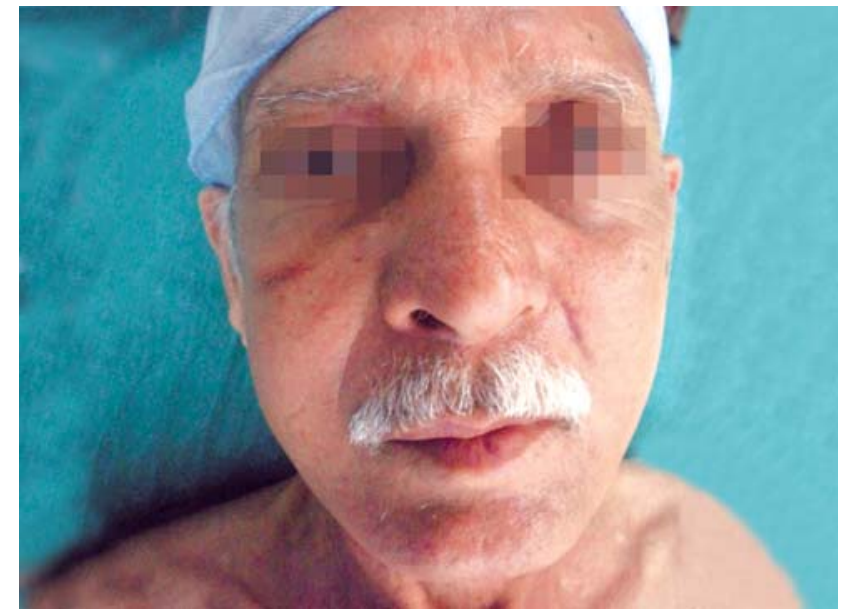

Fig. 1: Preoperative profile

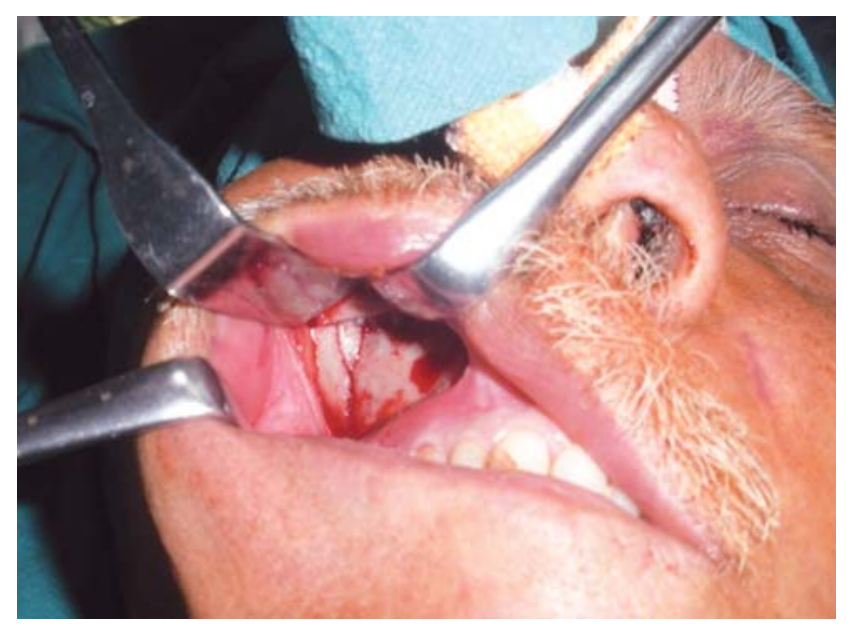

Fig. 3: Right side fracture exposed

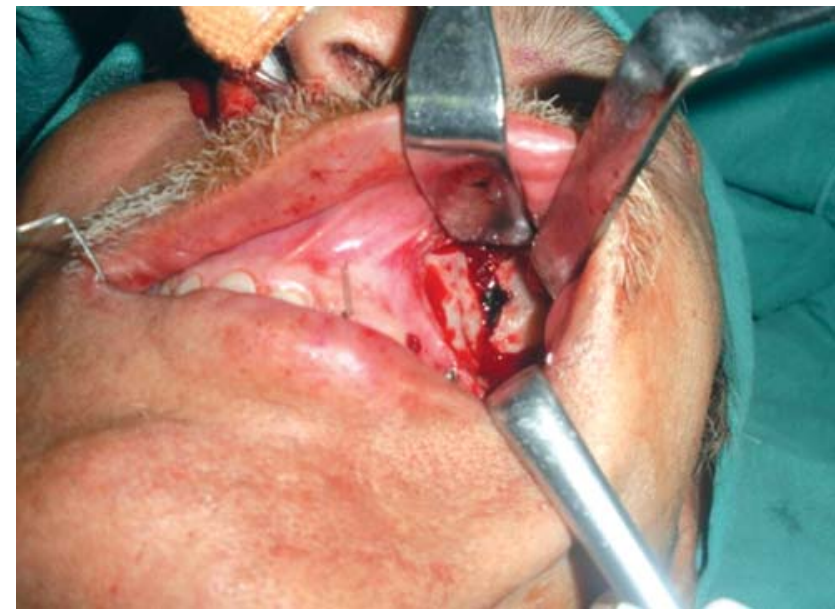

Fig. 5: Left side fracture exposed

rims. Preauricular, infraorbital and maxillary vestibular incisions can be performed when necessary.

\section{NASOETHMOID FRACTURES}

High energy frontal blow at the level of the nasal bone causes nasoethmoid fracture. Naso-orbito-ethmoidal facture can occur either in isolation or in association with other midfacial fractures. There is collapse and telescoping of

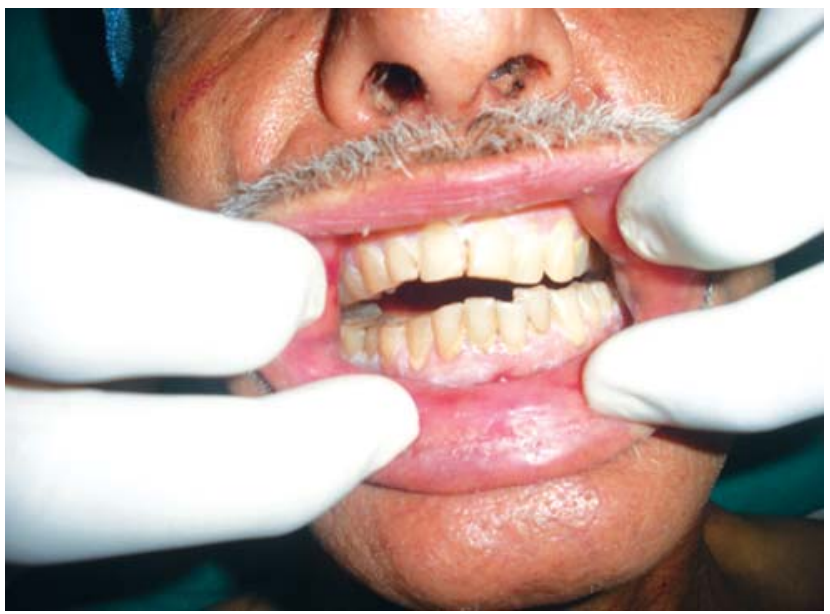

Fig. 2: Preoperative occlusion

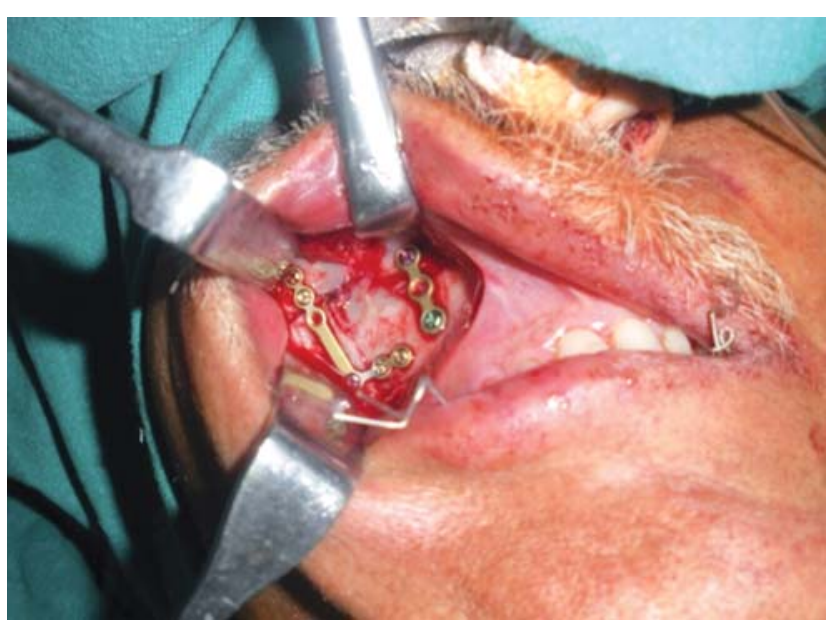

Fig. 4: Fracture reduced and fixed

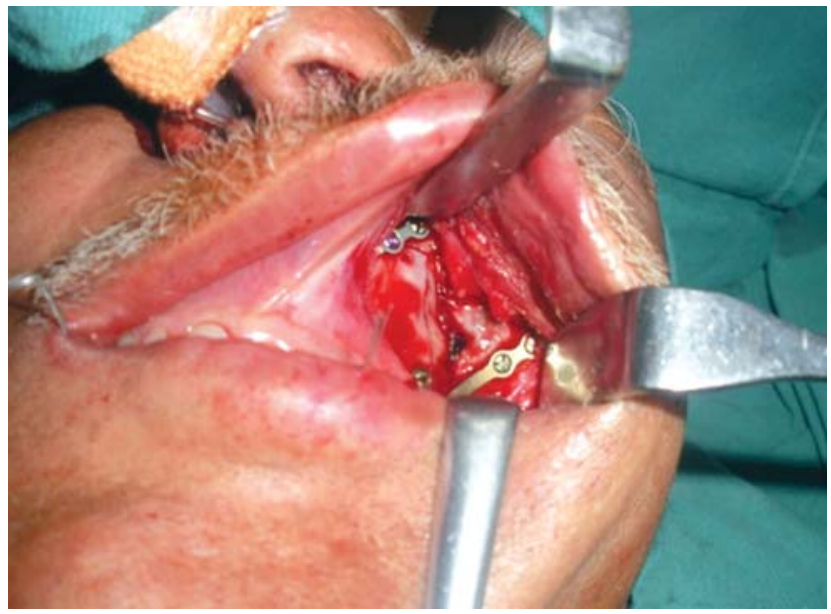

Fig. 6: Fracture reduced and fixed

the nasal bones and may be a fracture of the cribriform and fovea ethmoidalis. CSF leaks should be suspected. Traumatic telecanthus results from the disruption of the medial canthal tendon. Inadequate treatment leads to permanent telecanthus, flattened nasal bridge and rounding of the palpebral fissure. ${ }^{6}$ If the medial canthal ligament has been avulsed form the bone a transnasal canthopexy is performed or a microplate can be secured to the remnants of stable bone and the tendon 
secured to it with permanent suture. Nasal bridge augmentation can be carried out with a calvarial bone or Rib graft. Access to the area can be achieved through medial canthal incisons that can be connected in an $\mathrm{H}$ to create an open sky approach. Alternatively, the coronal approach can be extended over this area.

\section{ZYGOMATIC COMPLEX FRACTURES}

Zygomatic bone fracture is the second most common mid facial injury, following nasal fracture. The zygomatic complex fracture is termed as tripod fracture which is a misnomer as it is really a quadrapod fracture, as a result of disarticulation of the zygoma with the frontal, maxillary, sphenoid and temporal bones. These fractures typically result from a forces directed posteromedially impacting on the malar eminence. The fractures interconnect and are usually through the frontozygomatic suture and lateral orbit, across the zygomatic buttress, the inferior orbital rim and floor, and the lateral maxi llary wall. The lateral orbital fracture connects to the inferior orbital fracture through the inferior orbital fissure. Often these intraorbital components are non- or minimally displaced and difficult to detect on CT scan. The arch may be fractures in more than one place, leading to a free depressed segment. Because of their complex three-dimensional configuration, zygomatic fractures are notoriously difficult to completely reduce and fix in position while maintaining reduction. Because of the masseter pull on the zygomatic arch, these fractures have a propensity to become displaced with inadequate fixation.

Biomechanical analysis has been performed to evalate the most efficacious way of repairing these fractures. As noted above, Davidson, Rhinehart, and O’Hara have probably done the most logical studies, but controversy still exists. ${ }^{7}$ Despite their studies demonstrating the superiority of rigid fixation involving at least two sites, the prevailing method utilized in a survey of over 200 surgeons in Great Britain was the Gillies incision with FZ rigid fixation only. While some have apparently achieved good results with these methods, the evidence suggests that this may not consistently work. The FZ suture line is the least useful site to detect the degree of rotation of the fracture because many rotated and displaced fractures will not appear significantly displaced at the FZ. Additionally the stability of a single plate at the FZ is questionable under dynamic loads. The importance of proper repair can be seen in patients who
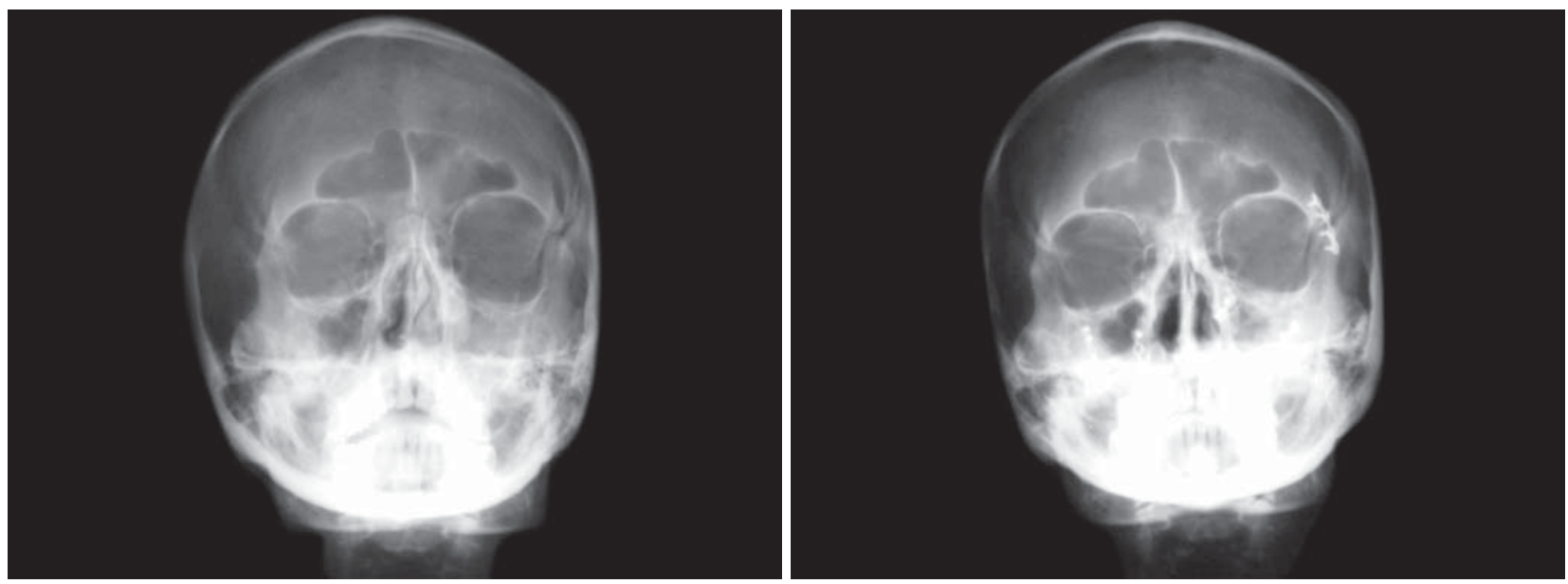

Fig. 7: Pre- and postoperative radiographs

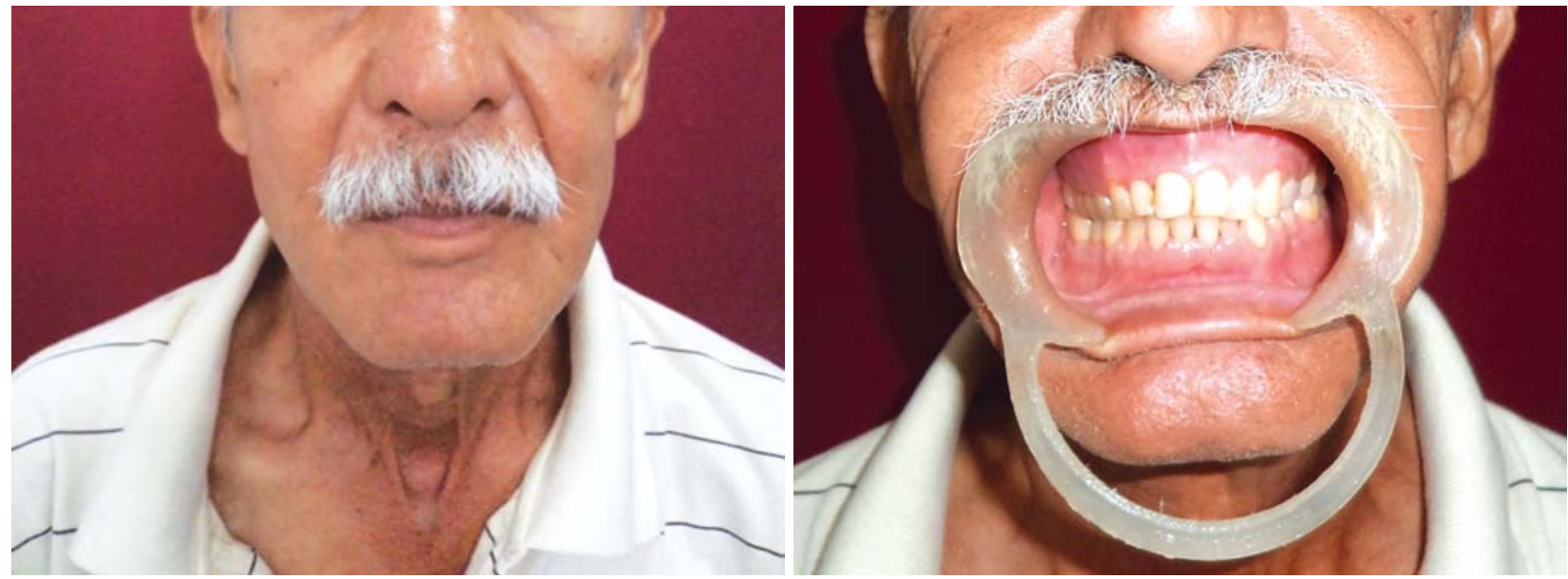

Fig. 8: Postoperative profile and occlusion 
have undergone insufficient reduction and/or fixation of the zygomatic complex fracture. They typically have flattening of the malar eminence, facial widening on the affected side, and frequently have unequal pupillary height with enophthalmos secondary to inadequately treated orbital wall fractures causing increased orbital volume.

If open reduction/fixation is to be done, good visualization is necessary to ensure adequate anatomic reduction

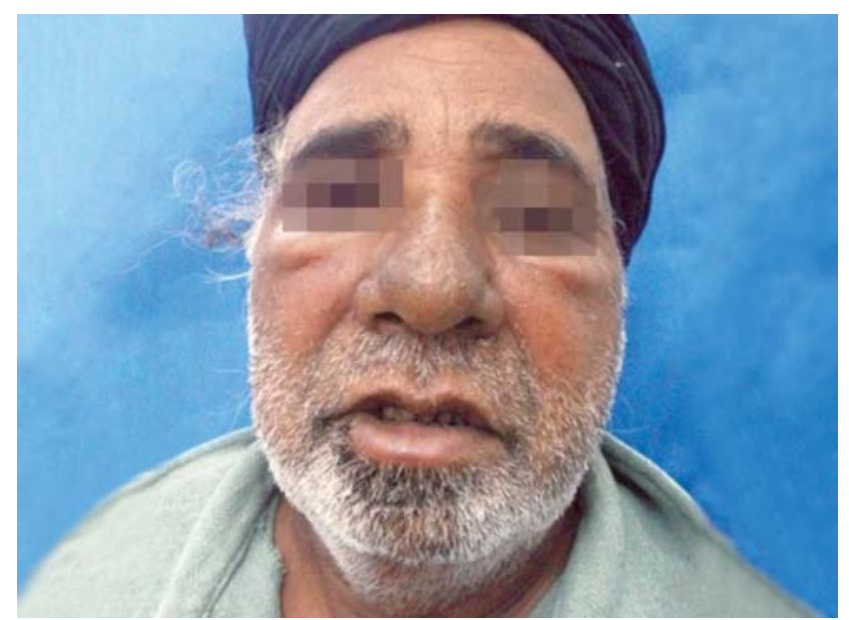

Fig. 9: Preoperative profile

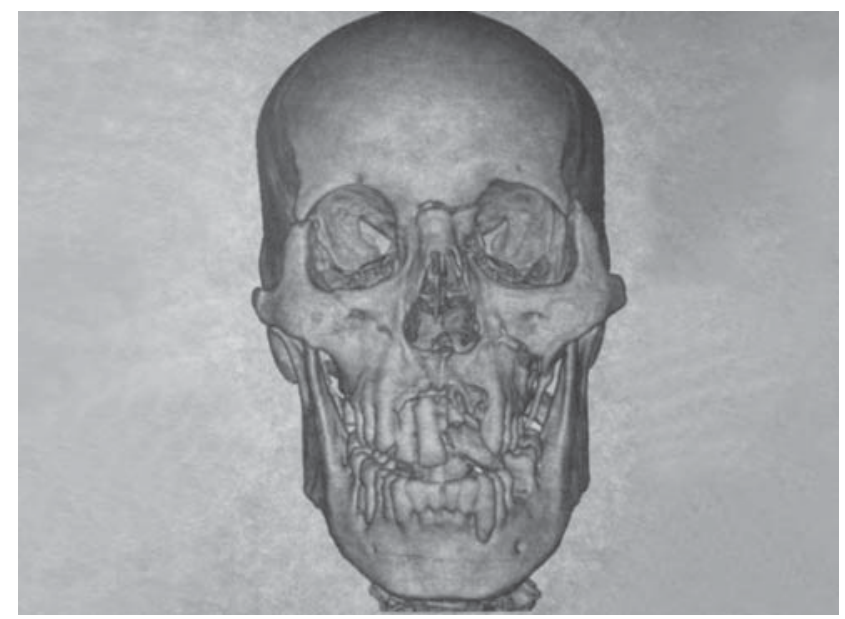

Fig. 10: Preoperative 3D CT scan

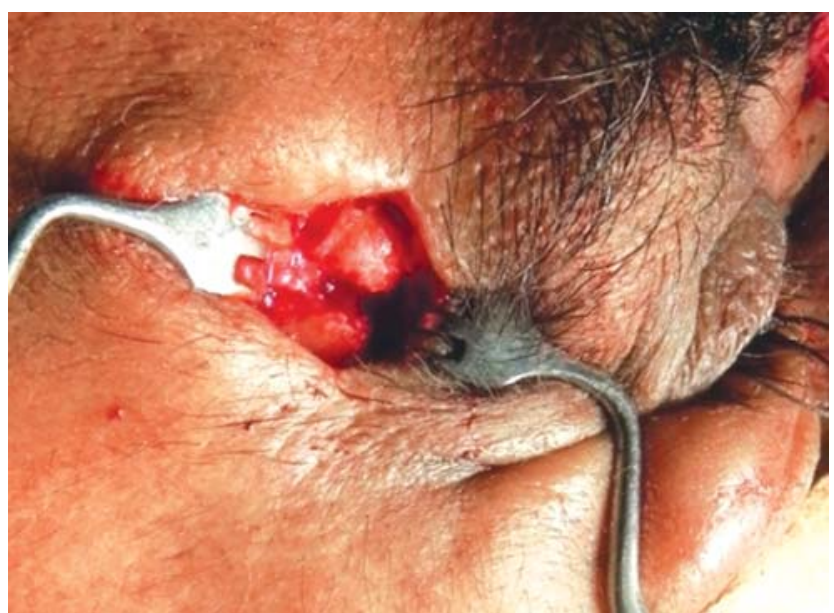

of displaced fractures. Multiple approaches are usually necessary, most often incorporating labiobuccal, transconjunctival (or subciliary), lateral brow incisions and or Gillies incisions. Coronal and hemicoronal approaches can be used, especially if fixation of the zygomatic arch is planned. This is a slightly controversial point as some authors insist that reduction and fixation of the arch is the key to obtaining a good result in cases where there is a displaced arch fracture. Gruss $^{8}$ has written extensively about these injuries and is a major proponent of this technique. He stresses the need for exploration and reduction/fixation based on late postoperative asymmetries after craniofacial procedures wherein other areas of rigid fixation were relied on to maintain midfacial projection, and the arch was reduced but not fixed. This seems to contradict results of some biomechanical studies.

\section{ORBITAL FRACTURES}

Isolated orbital fracture is not a common type of midfacial fracture, but the incidence of midfacial fractures involving the orbit is high because all Le Fort II and III fractures and those of the naso-orbito-ethmoidal and zygomaticomaxillary complexes involve orbital injury. Zygomaticomaxillary complex fracture is the most common fracture type with orbital involvement. Isolated fractures of the orbit often occur as a result of direct force to the globe of the eye. A sudden increase in intraorbital pressure creates an outward force that causes fracture of the weakest bony structures in internal orbital walls. Isolated orbital fractures can be classified as 'blow-out' or 'blow-in'. Most blow-out fractures affect the anteroinferomedial aspect of the orbital cavity and displace the orbital globe posteromedially and inferiorly. A significant increase in the volume of the orbital cavity results in enophthalmos of the globe. Herniation of the orbital roof and globe to the maxillary sinus occurs in such fractures. When an isolated fracture is caused by low-energy force, linear fracture of the orbit may be detected. Linear fractures

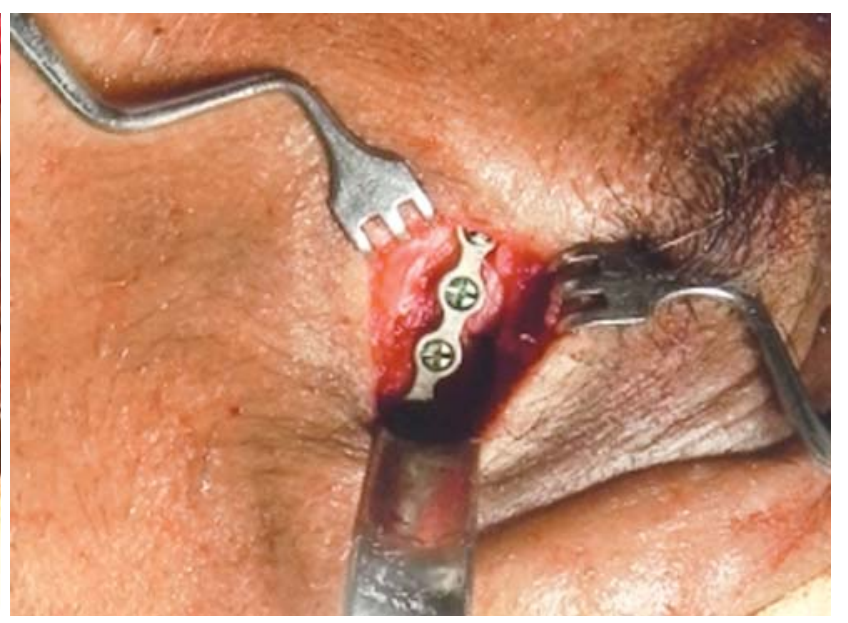

Fig. 11: Exposure, reduction and fixation of right $F Z$ fracture 

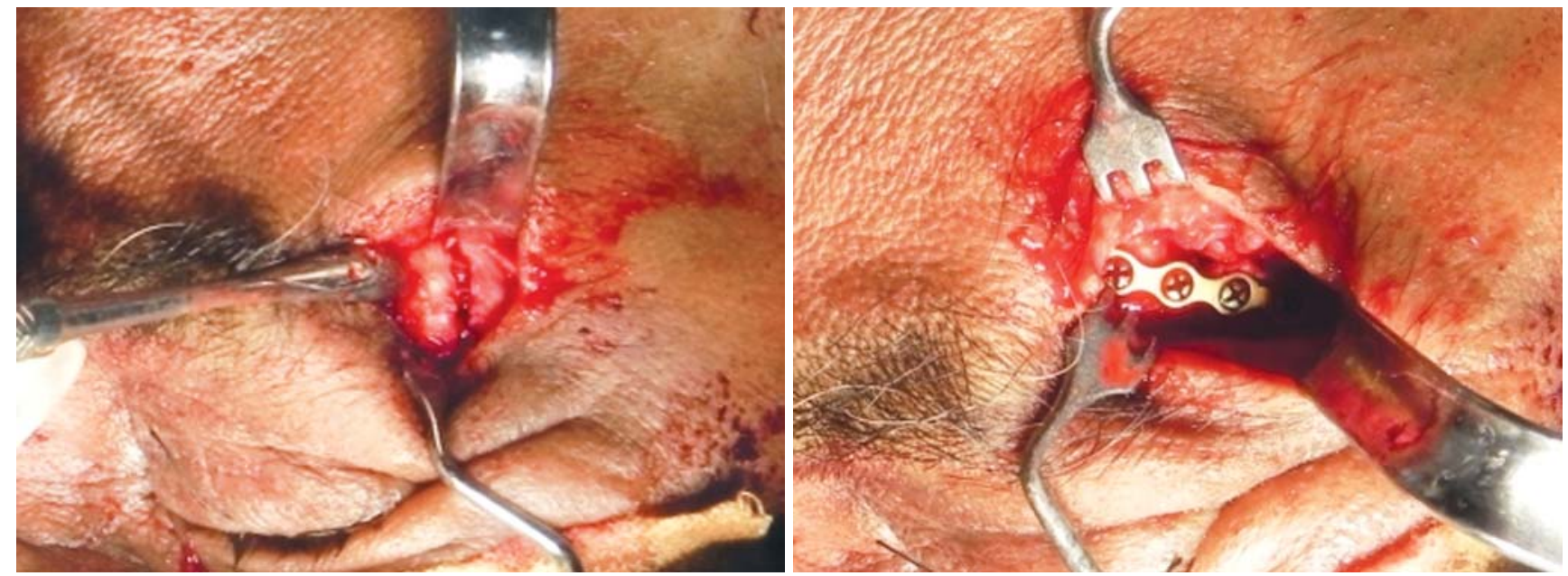

Fig. 12: Exposure, reduction and fixation of left FZ fracture

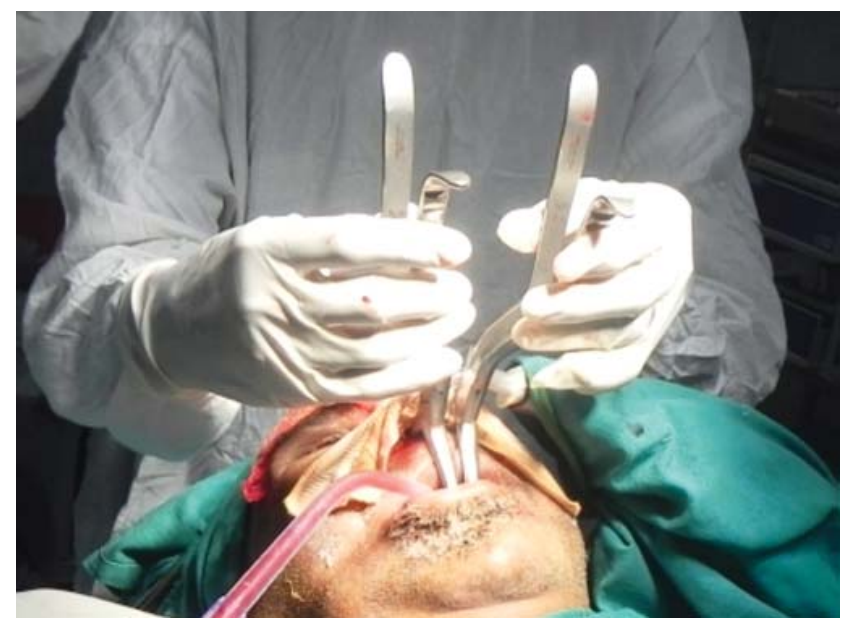

Fig. 13: Mobilization of maxilla

retain periosteal attachments and do not cause orbital globe herniation. More severe trauma causes a complex fracture involving two or more orbital walls. In complex internal orbital fractures, the globe is often displaced posteriorly and the optic canal may be involved. Blow-in fractures affect the orbital roof and may be diagnosed after severe injury of the anterior skull base. Rupture of the orbital roof reduces the orbital volume and often causes anteroinferior globe displacement.

The affected region should be inspected carefully to identify the presence of edema, chemosis, ecchymosis, lacerations, ptosis, asymmetric lid drape, canalicular injury, and/ or canthal tendon disruption. Any step deformity or mobility around the orbital rim should be palpated before edema develops in surrounding tissues. Neurosensation of the infraorbital and supraorbital nerves should be tested. Ophthalmologic consultation is very important and necessary. Limitation of ocular movements can be caused by mechanical entrapment or neurologic injury. Three-dimensional CT and magnetic resonance imaging are preferred for the evaluation of orbital fractures. Waters' projection is the most useful plain radiographic modality because it enables visualization of the orbital floor and roof. Ophthalmic ultrasonography and color Doppler imaging can provide additional information.

Subciliary and transconjunctival incisions are the most esthetically acceptable approaches to the orbital floor. Linear injuries of the orbital floor require no intervention unless they show signs of soft-tissue entrapment in fractured but selfreduced sites. In patients with blow-out or blow-in fractures, soft- and hard-tissue reduction and reconstruction are necessary. Grafting of the injured site with autografts, allografts, or alloplastic materials may be necessary to achieve proper anatomic reduction and stability and to prevent soft-tissue contraction. The Split calvarial, iliac crest and nasal septal cartilage are the best donor sites for autografts, and the use of alloplastic titanium mesh can be successful in cases requiring extra support.

Most internal orbital fractures cause volumetric contraction or expansion of the orbital cavity, which may lead to diplopia, enophthalmos, exophthalmos, proptosis, and/or extraocular muscle imbalance. Extraocular muscle imbalance and diplopia can be the result of extraocular muscle entrapment or neuropathy of the 3rd to 5th cranial nerves. An increase in orbital volume causes enophthalmos, which may occur weeks or months after injury. For some challenging fractures of the orbital floor, the transconjunctival approach may be safer than other methods. The placement of a transconjunctival incision at the conjunctival fornix appears to minimize the risk of eyelid malposition. A transantral endoscopic approach is an alternative method that avoids potential damage caused by lower-lid incisions.

\section{CONCLUSION}

Midface fractures are most often seen in young adult males. Children have a low incidence of midfacial fractures; probably due to the relatively larger cranial size, and the lack of complete maxillary sinus pneumatization. Intracranial injuries, CSF leaks, and ocular injuries must be suspected and ruled out in patients with facial injuries. There is little 


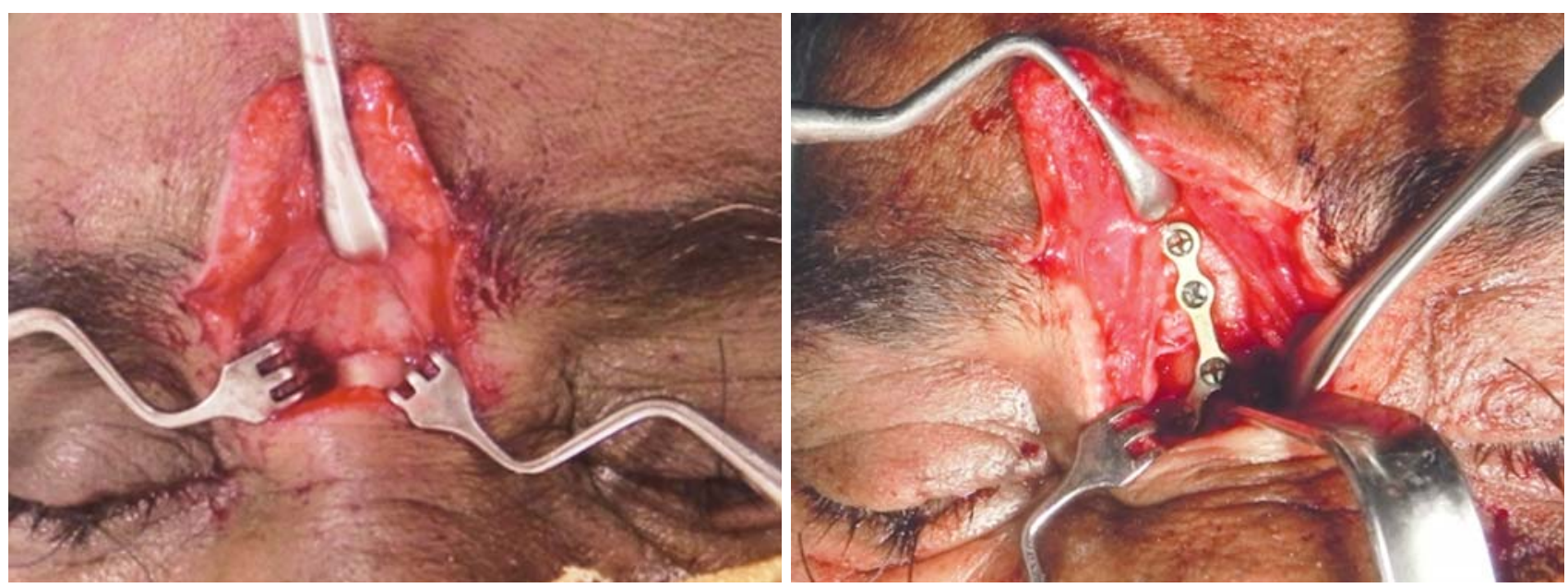

Fig. 14: Exposure, reduction and fixation of FN fracture

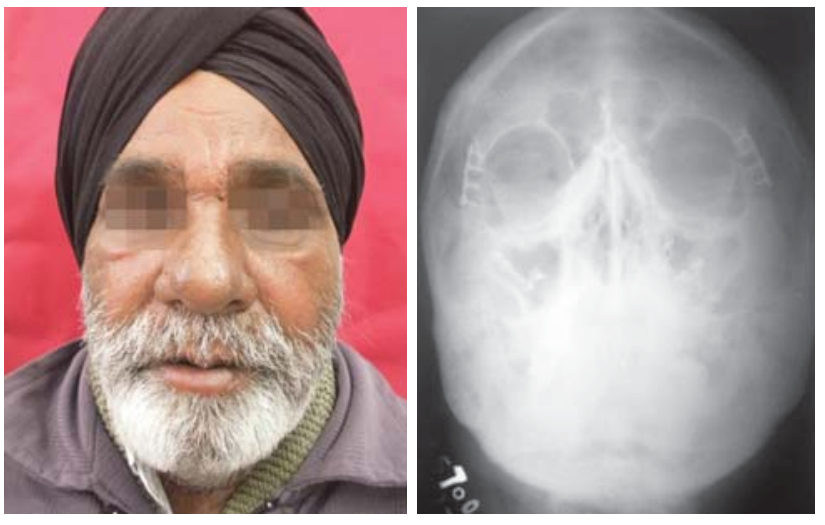

Fig. 15: Postoperative profile and radiograph

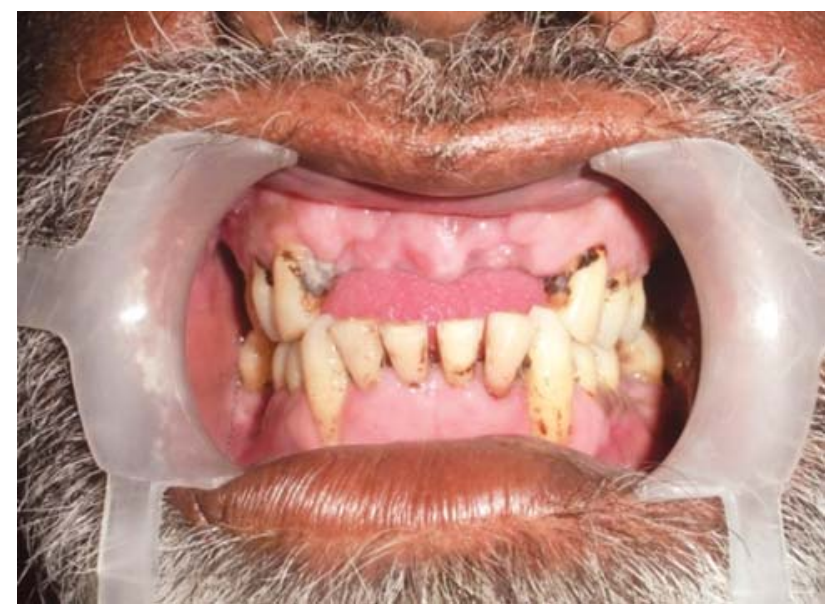

Fig. 16: Postoperative occlusion

difference in the outcome of stable patients whose fractures are treated very early versus those treated in a more delayed fashion. Rigid and semirigid plating systems perform well with few complications when used to repair midfacial fractures provided proper reduction is obtained. The dental occlusion must be used as the lower frame of reference when treating fractures that result in instability of the maxillary alveolus relative to the skull base. The most common complication after rigid repair of facial fractures appears to be malunion secondary to inadequate reduction. Bone grafting should be considered in the repair of comminuted fractures that result in gaps of $>5 \mathrm{~mm}$ between segments. Membranous bone is generally a better substrate for bone grafting in the face.

\section{REFERENCES}

1. Motamedi MHK. An assessment of maxillofacial fractures: a 5-year study of 237 patients. J Oral Maxillofac Surg 2003;61: 61-64.

2. Cook HE, Rowe M. A retrospective study of 356 midfacial fractures occurring in 225 patients. J Oral Maxillofac Surg 1990;48: 574-578.

3. Mukerji R, Mukerji M, McGurk M. Mandibular fractures: historical perspective. Br J Oral Maxillofac Surg 2006;44:222-228.

4. Le Fort R. Etude experimentale sur les fractures de la machoire superiore. Rev Chir 1901;23:208-227.

5. Gillies HD, Kilner TP, Stone D. Fractures of the malar-zygomatic compound: with a description of a new X-ray position. Br J Surg 1927;14:651-656.

6. Markowitz BL, Manson PN, Sargent L, et al. Management of medial canthal tendon in nasoethmoid orbital fractures: the importance of the central fragment in classification and treatment, Plast Reconstr Surg 1991;87:843-853.

7. Davidson J, Nickerson D, Nickerson B. Zygomatic fractures: comparison of methods of internal fixation. Plast Reconstr Surg 1990 July;86:25-32.

8. Gruss JS, Van Wyck L, Phillips JH, Antonyshyn O. The importance of the zygomatic arch in complex midfacial fracture repair and correction of post-traumatic orbitozygomatic deformities. Plast Reconstr Surg 1990 Jun;85(6):878-889. 